\title{
Plasma isoflavone concentration is associated with decreased risk of type 2 diabetes in Korean women but not men: results from the Korean Genome and Epidemiology Study
}

\author{
Kwang-Pil Ko • Cheong-Sik Kim • Younjhin Ahn • \\ Seon-Joo Park • Yeon-Jeong Kim • Jae Kyung Park • \\ Young-Khi Lim $\cdot$ Keun-Young Yoo $\cdot$ Sung Soo Kim
}

Received: 16 June 2014 / Accepted: 5 November 2014 / Published online: 23 December 2014

(C) Springer-Verlag Berlin Heidelberg 2014

\begin{abstract}
Aims/hypothesis To examine the association between soybean products and risk of type 2 diabetes, we measured four isoflavone biological markers-genistein, daidzein, glycitein and equol-in a nested case-control study.

Methods The study population was composed of 693 cases (316 women and 377 men) and 698 matched controls (317 women and 381 men) within the Korean Genome and Epidemiology Study. The concentrations of isoflavone biomarkers were measured using HPLC-MS/MS on plasma samples that were collected at baseline. A stratified analysis was undertaken to examine the association between plasma isoflavone concentrations and risk of type 2 diabetes according to sex and equol production. Logistic regression models were used to compute ORs and $95 \%$ CIs adjusted for confounders.
\end{abstract}

\section{K.-P. Ko}

Department of Preventive Medicine, Gachon University, Incheon, Korea

K.-P. Ko • C.-S. Kim • S.-J. Park • Y.-J. Kim • J. K. Park •

S. S. Kim $(\bowtie)$

Division of Epidemiology and Health Index, Center for Genome

Science, Korea Centers for Disease Control \& Prevention, 187

Osongsaengmyeong 2-ro, Osong-eup, Cheongju-si,

Chungcheongbuk-do 363-951, Korea

e-mail: ksungsoo@korea.kr

\section{Y. Ahn}

Division of Cardiovascular and Rare Disease, Center for Biomedical Diseases, Korea Centers for Disease Control \& Prevention, Osong, Korea

\section{Y.-K. Lim}

Department of Radiological Science, Gachon University, Incheon, Korea

K.-Y. Yoo

Department of Preventive Medicine, Seoul National University

College of Medicine, Seoul, Korea
Results In women, compared with the lowest quartile of plasma concentration of genistein, the highest quartile exhibited a significantly decreased risk of diabetes (OR $0.58,95 \% \mathrm{CI}$ $0.35,0.95)$. When stratified by equol-producing status in women, the OR for diabetes in the highest vs the lowest quartile of genistein concentration was 0.31 (95\% CI 0.16 , $0.60)$ in equol producers, but genistein concentration was not associated with risk of diabetes in equol non-producers ( $p$ for interaction $=0.013$ ). In men, isoflavone concentrations were not associated with risk of diabetes, regardless of equolproducing status.

Conclusions/interpretation High plasma concentrations of genistein were associated with a decreased risk of type 2 diabetes in women. This inverse association was prominent in equol-producing participants. These results suggest a beneficial effect of a high intake of soybean products on risk of type 2 diabetes in women.

Keywords Equol $\cdot$ Genistein $\cdot$ Isoflavone $\cdot$ Type 2 diabetes

Abbreviations
$\begin{array}{ll}\text { FFQ } & \text { Foods frequency questionnaire } \\ \text { FSG } & \text { Fasting serum glucose } \\ \text { KoGES } & \text { Korean Genome and Epidemiology Study }\end{array}$

Introduction

The prevalence of type 2 diabetes is rapidly increasing worldwide in parallel with an ageing population, economic growth and the urbanisation of developing countries. The number of patients with diabetes was estimated to be 171 million in 2000 and is projected to rise to 366 million in 2030, contributing to the so-called 'diabetes epidemic' [1]. This diabetes epidemic is caused by lifestyle factors such as physical inactivity, 
obesity and the Westernisation of dietary habits; therefore the identification of protective factors associated with type 2 diabetes is important for establishing prevention strategies.

Previous studies have suggested that endogenous sex hormones may have a role in a sex-dependent development of type 2 diabetes by influencing body weight, body fat distribution and insulin sensitivity via effects on adiposity [2]. Soybean products are a candidate food that may prevent type 2 diabetes because the chemical structure of isoflavones from soybeans is similar to that of oestrogen. In addition to the fact that isoflavones can regulate glucose homeostasis via oestrogen-like actions, soybeans have a glucose-lowering effect through a modulation of insulin resistance, an inhibition of intestinal glucose uptake and the prevention of glucoseinduced lipid peroxidation [3-5]. However, because the doses of isoflavone from soy products used in in vitro or animal studies are much higher than those in the soy products regularly consumed by humans, the health benefits of soy for type 2 diabetes have been found in epidemiological studies to be limited and inconsistent [6-9]. Although there is emerging evidence that the response to isoflavone intake may vary according to an individual's equol-producing capacity, previous studies have not considered equol, which is a metabolite of isoflavone [10, 11].

The inconsistent results regarding the dietary intake of soy products may be due to the use of food frequency questionnaires (FFQs), a low amount of soy consumption according to ethnicity and interindividual variability in the response to isoflavones according to equol-producing. Although we can estimate the usual intake of soy products with FFQs, FFQs are vulnerable to measurement errors because of memory decay. In addition, when using FFQs, we cannot know how much of the soy intake will reach the systemic circulation and we cannot assess the individual's equol-producing capacity. Thus, the measurement of biological markers and a prospective cohort study design in a population with high soybean consumption can provide valid results to assess the association between the intake of soybean products and type 2 diabetes [12].

Because isoflavones are abundant in soybeans, plasma isoflavone concentrations reflect the dietary intake of soybean products [13]. In this Korean nested case-control study, we measured isoflavone biological markers (genistein, daidzein, glycitein and equol) to examine whether isoflavones are associated with the development of type 2 diabetes according to sex and equol-producing status.

\section{Methods}

Study population and data collection In 2001, the Korea Centers for Disease Control and Prevention launched a cohort project under the name of Korean Genome and Epidemiology
Study (KoGES) to identify environmental and genetic risk factors (and their interactions) that contribute to the development of major chronic diseases, such as hypertension, type 2 diabetes and the metabolic syndrome.

Individuals who were eligible to participate in this study were selected from an ongoing population-based cohort of KoGES in a rural community (Ansung) and an urban community (Ansan). Detailed information on the procedure and design for the Ansung and Ansan cohorts has previously been reported [14, 15]. Briefly, in 2000, the size of the base population of individuals aged from 40 to 69 years of age was 132,906 for Ansung and 554,998 for Ansan. To select a representative sample from each community, five Myeons (governing regions similar to townships) in Ansung were selected out of 11 Myeons by cluster sampling, and 5,018 people were recruited from the 7,192 eligible individuals (giving a response rate $69.8 \%$ ); the telephone directory was used to make a random selection in Ansan. Among 21 Dongs (governing regions similar to districts) in Ansan as the primary sampling units, 400 randomly selected telephone numbers in each Dong were assigned to the sub-sampling unit. A total of 5,020 people participated after contact had been made with 10,957 individuals by telephone call (a response rate of $45.8 \%$ ). A total of 10,038 men and women were recruited from 2001 through 2002, and a baseline study was conducted.

Information on the general characteristics of the participants' medical history, lifestyle, physical activity level, diet, reproductive factors and psychosocial factors was obtained through interviews using a structured questionnaire. Information on diet was collected using an FFQ developed by the Korean National Institutes of Health. A validation test had previously been conducted for this FFQ [16]. The FFQ included 103 food items to assess the usual dietary intake consumed during the preceding 12 months, and dietary isoflavone intake was calculated using the isoflavone database for usual Korean foods [17]. However, there was no information about the validity of the dietary assessment to estimate isoflavone intake using the FFQ.

Anthropometric measurements such as blood pressure, height, weight, waist circumference and body composition were also obtained. At the baseline when the study population was enrolled, plasma was immediately separated by centrifugation $\left(382 \mathrm{~g}\right.$ for $20 \mathrm{~min}$ at $4^{\circ} \mathrm{C}$ ) for clinical laboratory measurements such as fasting serum glucose (FSG), total cholesterol, triacylglycerols and HDL-cholesterol using a Hitachi 747 chemistry analyser (Hitachi, Tokyo, Japan). After 8$14 \mathrm{~h}$ of overnight fasting, all participants underwent a $2 \mathrm{~h}$ $75 \mathrm{~g}$ OGTT. Blood and spot urine samples were collected, and serum, plasma and buffy coat samples were stored at $-70^{\circ} \mathrm{C}$ or in an LN2 tank. The study participants gave their informed consent, and the study protocol was approved by the institutional review board of the Korea Centers for Disease Control and Prevention. 
Follow-up and selection of cases and controls Follow-up examinations were conducted at 2 year intervals. The follow-up rates were $85.7 \%, 74.9 \%$ and $66.6 \%$, respectively, at the 2, 4 and 6 year follow-up surveys after the baseline survey.

At each follow-up examination, participants were interviewed using a questionnaire, and their FSG levels were measured. In addition, their serum glucose levels were measured $2 \mathrm{~h}$ after an OGTT in the same manner as for the baseline survey. A participant was considered to be an incident case if at least one of the following criteria was met: FSG levels $\geq 7.0 \mathrm{mmol} / \mathrm{l}$, serum glucose levels after a $2 \mathrm{~h}$ OGTT $\geq 11.1 \mathrm{mmol} / \mathrm{l}$ or the individual self-reporting treatment with a hypoglycaemic medication.

In Ansung, plasma samples were collected from 2003 (at the first follow-up examinations), and we considered the first follow-up examinations as the baseline. As of December 2008, we had identified 700 newly diagnosed incident individuals with type 2 diabetes via an active bi-annual follow-up. We conducted frequency matching with cases to select controls within the cohort based on age, sex and area of residence (Ansung or Ansan). The the controls were free of diabetes and alive at the time of diagnosis of the matched cases.

Measurements of plasma concentrations of genistein, daidzein, glycitein and equol We measured the plasma concentrations of the isoflavones genistein, daidzein, glycitein and equol, a daidzein metabolite, using liquid chromatography and MS, which allow precise, sensitive and rapid analysis with limited volumes of plasma [18].

For quality control, we calculated the coefficients of variation (i.e. the SD divided by the mean multiplied by 100 to result in a percentage) within each batch using 141 replicated samples for genistein, glycitein, daidzein and equol. In our study, the mean coefficients of variation were $6.8 \%$ for genistein, $10.1 \%$ for glycitein, $8.9 \%$ for daidzein and $8.2 \%$ for equol.

Statistical analysis A $t$ test, Wilcoxon's rank sum test or $\chi^{2}$ test was used to identify differences between cases and controls with regard to the means or proportions of baseline characteristics such as age, sex, cigarette smoking, alcohol consumption, BMI, systolic blood pressure, total energy intake and isoflavone intake measured by FFQ. The concentrations of the isoflavones was divided into quartiles within the control groups. Unconditional logistic regression models were used to assess the associations between concentration of isoflavones and type 2 diabetes in men and women, and ORs and $95 \%$ CIs for risk of type 2 diabetes were calculated adjusted for age (continuous variable), area of residence (Ansung/Ansan), cigarette smoking (never/past/current smoking), alcohol drinking (never/past/current drinking), BMI (continuous variable), systolic BP (continuous variable) and FSG (continuous variable) at baseline, all of which are known risk factors for type 2 diabetes and are associated with isoflavone levels. The $p$ values for the trend test were calculated by treating the numerical values of the categorical variable as a score in multiple logistic regression models. A stratified analysis was completed to examine the association between the plasma genistein, daidzein and glycitein concentrations and the risk of type 2 diabetes according to sex and equol-producing status. The $p$ values for interaction were calculated by adding an interaction term to the logistic regression model. We defined equol non-producers as individuals who had plasma concentrations below the limit of detection of the present assay, $0.068 \mathrm{ng} / \mathrm{ml}$, as in previous studies [19-21]. All analyses were conducted using SAS 9.2 (SAS Institute, Cary, NC, USA).

\section{Results}

After excluding participants in whom a plasma isoflavone concentration could not be measured, 693 individuals with type 2 diabetes and 698 controls were included in the final analysis. The demographic and lifestyle characteristics of the participants with type 2 diabetes and the matched controls are presented in Table 1. The mean age of the cases and control participants was 53.8 and 53.7 years for women, and 51.3 and 51.3 years for men, respectively. The BMI and systolic BP were higher in cases than controls. There were no significant differences in the selected characteristics, including age, sex, area of residence, history of cigarette smoking, alcohol consumption history and dietary intake between the cases and the controls.

In women, the median plasma equol concentration was lower in the cases than in the controls $(5.1 \mathrm{ng} / \mathrm{ml}$ for cases and $14.8 \mathrm{ng} / \mathrm{ml}$ for controls; $p=0.013$; Table 2 ). There were no significant differences in the median plasma concentration between cases and controls for the other isoflavones tested. In men, the median plasma concentration of daidzein was higher in the cases than in the controls $(114 \mathrm{ng} / \mathrm{ml}$ for cases and $83.9 \mathrm{ng} / \mathrm{ml}$ for controls; $p=0.038$ ).

In women, a decreasing trend in the risk of type 2 diabetes was demonstrated with categories of increasing genistein concentration ( $p$ value for trend $=0.024)$, and a significantly decreased risk was found in women with the highest concentration of genistein (compared with the lowest quartile: OR 0.58, 95\% CI 0.35, 0.95; Table 3). Compared with the lowest quartile, the highest quartile of equol concentration was marginally associated with a decreased risk of type 2 diabetes in women (OR $0.67,95 \%$ CI $0.43,1.06$ ). There was no association between the risk of type 2 diabetes and the plasma concentration of daidzein and glycitein. In men, the concentrations of the four isoflavones genistein, glycitein, daidzein 
Table 1 Baseline characteristics of the participants with type 2 diabetes and the controls

\begin{tabular}{|c|c|c|c|c|c|c|}
\hline \multirow[t]{2}{*}{ Variable } & \multicolumn{3}{|l|}{ Women } & \multicolumn{3}{|l|}{ Men } \\
\hline & Cases $(n=316)$ & Controls $(n=317)$ & $p$ value & Cases $(n=377)$ & Controls $(n=381)$ & $p$ value \\
\hline Age $(\text { years })^{\mathrm{a}}$, mean $\pm \mathrm{SD}$ & $53.8 \pm 9.0$ & $53.7 \pm 8.8$ & 0.89 & $51.3 \pm 8.4$ & $51.3 \pm 8.3$ & 0.93 \\
\hline \multicolumn{7}{|l|}{ Area $^{\mathrm{b}}, n(\%)$} \\
\hline Ansung (4 years follow-up) & 97 (30.7) & $97(30.6)$ & \multirow{2}{*}{0.98} & $77(20.4)$ & $78(20.5)$ & \multirow[t]{2}{*}{0.99} \\
\hline Ansan (6 years follow-up) & $219(69.3)$ & $220(69.4)$ & & $300(79.6)$ & $303(79.5)$ & \\
\hline \multicolumn{7}{|l|}{ Smoking ${ }^{\mathrm{b}, \mathrm{d}}, n(\%)$} \\
\hline No history of smoking & $293(92.7)$ & 304 (95.9) & \multirow[t]{3}{*}{0.15} & $68(18.0)$ & $73(19.2)$ & \multirow[t]{3}{*}{0.92} \\
\hline Ex-smoker & $13(4.1)$ & $6(1.9)$ & & $162(43.0)$ & $160(42.0)$ & \\
\hline Current smoker & $10(3.2)$ & $6(1.9)$ & & $147(39.0)$ & $148(38.9)$ & \\
\hline \multicolumn{7}{|l|}{ Alcohol drinking ${ }^{\mathrm{b}, \mathrm{d}}, n(\%)$} \\
\hline No history of drinking & $219(69.3)$ & $232(73.2)$ & \multirow[t]{3}{*}{0.08} & $62(16.5)$ & $73(19.2)$ & \multirow[t]{3}{*}{0.53} \\
\hline Ex-drinker & $84(26.6)$ & $81(25.6)$ & & $280(74.3)$ & $277(72.7)$ & \\
\hline Current drinker & $13(4.1)$ & $4(1.3)$ & & $35(9.3)$ & $30(7.9)$ & \\
\hline BMI $\left(\mathrm{kg} / \mathrm{m}^{2}\right)^{\mathrm{a}}$, mean $\pm \mathrm{SD}$ & $25.9 \pm 3.3$ & $24.8 \pm 3.3$ & $<0.001$ & $25.0 \pm 2.8$ & $24.2 \pm 2.7$ & $<0.001$ \\
\hline Systolic $\mathrm{BP}^{\mathrm{b}}(\mathrm{mmHg})$, mean $\pm \mathrm{SD}$ & $121 \pm 19$ & $118 \pm 21$ & 0.06 & $122 \pm 18$ & $117 \pm 16$ & 0.001 \\
\hline \multicolumn{7}{|l|}{ Dietary intake per day ${ }^{c}$} \\
\hline Total energy $(\mathrm{kJ})$, mean $\pm \mathrm{SD}$ & $7,611 \pm 2,795$ & $7,640 \pm 2,342$ & 0.58 & $8,271 \pm 2,291$ & $8,437 \pm 3,020$ & 0.47 \\
\hline Protein $(\mathrm{g})$, mean $\pm \mathrm{SD}$ & $63.2 \pm 29.7$ & $61.9 \pm 20.4$ & 0.66 & $70.9 \pm 26.4$ & $70.8 \pm 29.9$ & 0.94 \\
\hline Fat $(\mathrm{g})$, mean $\pm \mathrm{SD}$ & $28.6 \pm 18.1$ & $28.9 \pm 15.5$ & 0.58 & $36.6 \pm 17.9$ & $37.0 \pm 21.4$ & 0.84 \\
\hline Carbohydrate (g), mean \pm SD & $322 \pm 113$ & $324 \pm 103$ & 0.87 & $335 \pm 86.9$ & $344 \pm 114$ & 0.19 \\
\hline Vitamin $\mathrm{C}(\mathrm{mg})$, mean $\pm \mathrm{SD}$ & $128 \pm 91.8$ & $119 \pm 98.4$ & 0.08 & $111 \pm 68.6$ & $111 \pm 68.2$ & 0.85 \\
\hline Fibre $(\mathrm{g})$, mean $\pm \mathrm{SD}$ & $20.6 \pm 9.3$ & $20.0 \pm 8.7$ & 0.53 & $20.1 \pm 8.5$ & $20.0 \pm 9.0$ & 0.97 \\
\hline Isoflavone $(\mathrm{mg})$, mean $\pm \mathrm{SD}$ & $18.7 \pm 13.1$ & $18.4 \pm 13.3$ & 0.53 & $16.6 \pm 12.1$ & $15.6 \pm 12.7$ & 0.16 \\
\hline
\end{tabular}

${ }^{a} p$ values were calculated by the $t$ test

${ }^{\mathrm{b}} p$ values were calculated by the $\chi^{2}$ test

${ }^{\mathrm{c}}$ Dietary intake was measured by the FFQ and $p$ values were calculated by Wilcoxon's rank sum test

${ }^{\mathrm{d}}$ There were missing data in smoking $(n=1)$ and alcohol drinking history $(n=1)$

and equol showed no association with risk of type 2 diabetes, except for the second highest quartile of genistein.

There was a significant interaction for risk of type 2 diabetes between genistein concentration and sex ( $p$ for interaction $=0.034$ ). There was no association between risk of type 2 diabetes and the dietary isoflavone intake measured by the FFQ in either men or women.
Among the women, $64.8 \%$ of the participants were equol producers (Table 4). When the female study participants were stratified by equol production, the results differed according to equol-producing status. In equol producers, the highest concentration of genistein was associated with a significantly decreased risk of type 2 diabetes (OR 0.31 , 95\% CI 0.16 , 0.60 ), and a dose-response relationship was also found

Table 2 Plasma concentration of isoflavones among cases and matched controls by sex

\begin{tabular}{|c|c|c|c|c|c|c|c|c|c|c|}
\hline \multirow{3}{*}{$\begin{array}{l}\text { Isoflavone } \\
\text { (ng/ml) }\end{array}$} & \multicolumn{5}{|l|}{ Women } & \multicolumn{5}{|l|}{ Men } \\
\hline & \multicolumn{2}{|l|}{ Cases $(n=316)$} & \multicolumn{2}{|l|}{ Controls $(n=317)$} & \multirow[t]{2}{*}{$p$ value $^{\mathrm{a}}$} & \multicolumn{2}{|l|}{ Cases $(n=377)$} & \multicolumn{2}{|l|}{ Controls $(n=381)$} & \multirow[t]{2}{*}{$p$ value $^{\mathrm{a}}$} \\
\hline & Median (IQR) & Mean \pm SD & Median (IQR) & Mean \pm SD & & Median (IQR) & Mean $\pm \mathrm{SD}$ & Median (IQR) & Mean \pm SD & \\
\hline Genistein & $209(112-405)$ & $337 \pm 385$ & $237(121-478)$ & $413 \pm 544$ & 0.181 & $290(135-528)$ & $428 \pm 456$ & $245(121-540)$ & $414 \pm 466$ & 0.144 \\
\hline Glycitein & $8.0(6.3-12.0)$ & $12.4 \pm 20.5$ & $8.0(6.2-11.8)$ & $10.6 \pm 8.5$ & 0.623 & $8.3(6.8-12.5)$ & $12.0 \pm 12.7$ & $8.3(6.7-11.4)$ & $11.1 \pm 10.4$ & 0.200 \\
\hline Daidzein & $73.4(27.1-161)$ & $180 \pm 753$ & $75.8(34.1-177)$ & $153 \pm 219$ & 0.358 & $114(46.2-207)$ & $185 \pm 297$ & $83.9(40.7-175)$ & $198 \pm 730$ & 0.038 \\
\hline Equol & $5.1(0.07-56.7)$ & $77.3 \pm 207$ & $14.8(0.07-95.0)$ & $97.0 \pm 292$ & 0.013 & $17.1(0.13-102)$ & $120 \pm 329$ & $13.8(0.07-86.3)$ & $85.0 \pm 176$ & 0.349 \\
\hline
\end{tabular}

${ }^{a} p$ values were calculated by Wilcoxon's rank sum test 


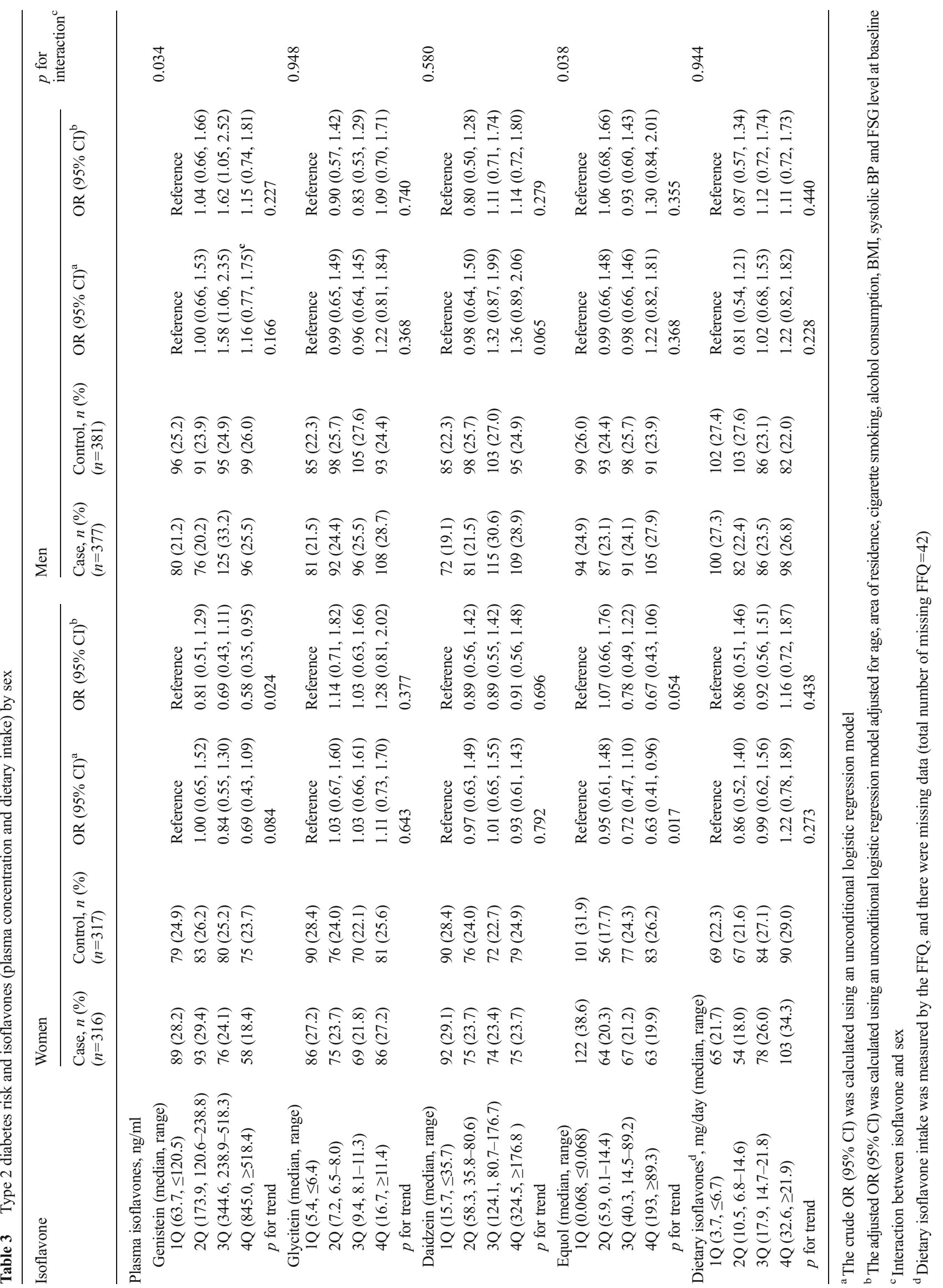




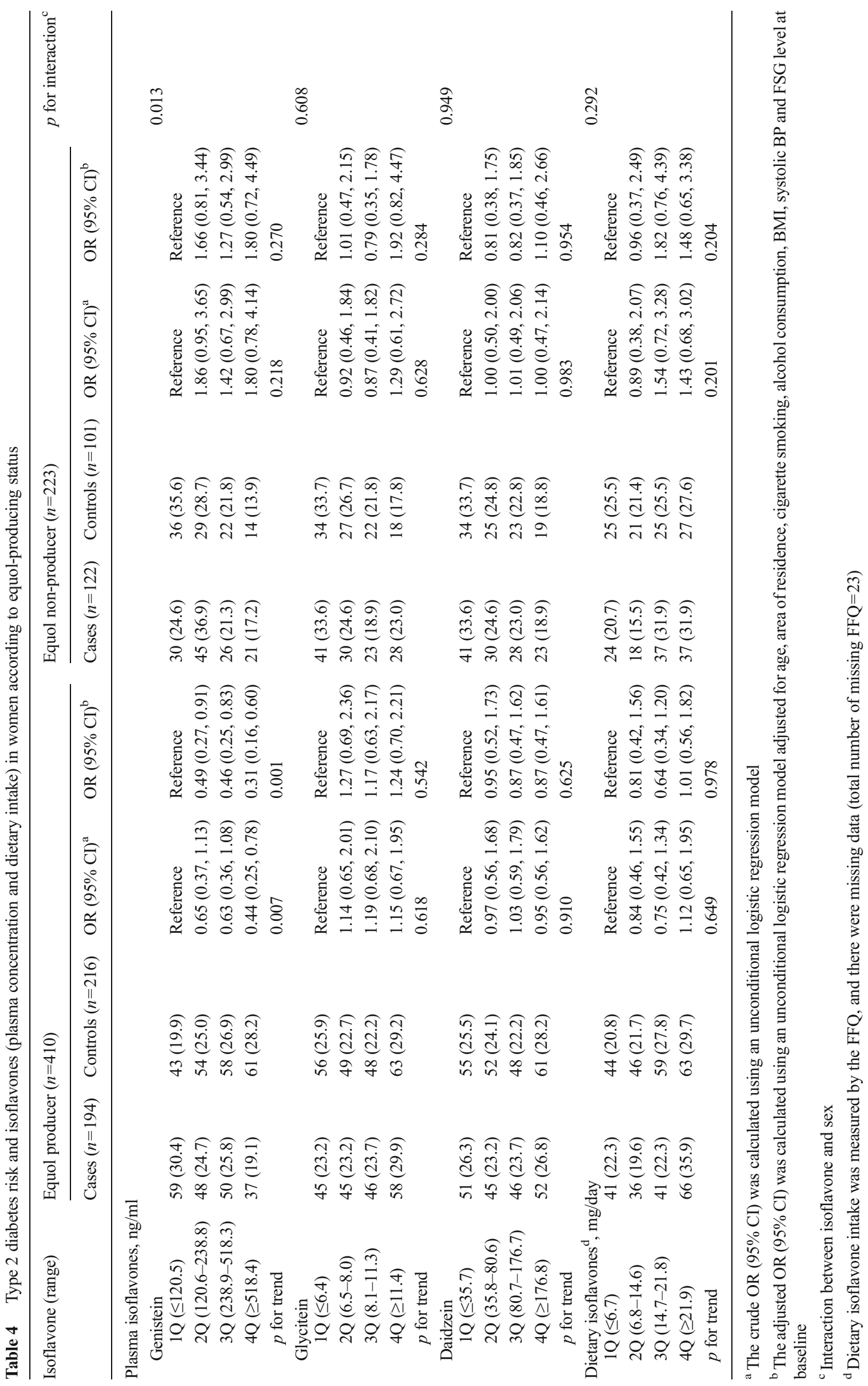


between the concentration of genistein and the risk of type 2 diabetes ( $p$ value for trend $=0.001$ ). However, in equol nonproducers, the genistein concentration was not associated with risk of type 2 diabetes. There was a significant interaction for risk of type 2 diabetes between the genistein concentration and the equol-producing status ( $p$ for interaction $=0.013$ ). The concentrations of daidzein and glycitein were not associated with risk of type 2 diabetes in women regardless of equolproducing status.

In men, no isoflavone was associated with risk of type 2 diabetes, regardless of equol-producing status (Table 5).

\section{Discussion}

In this nested case-control study using a population-based cohort in Korea, isoflavone biomarkers such as genistein were associated with a decreased risk of type 2 diabetes in women. The inverse association between genistein and type 2 diabetes was prominent in equol-producing individuals. However, there was no association between isoflavone and type 2 diabetes in men regardless of equol-producing status.

Oestrogen and oestrogen receptors are known to regulate glucose homeostasis by influencing glucose metabolism in the central nervous system, pancreatic beta cells, muscles, liver and adipocytes. Oestrogen increases insulin secretion in beta cells and glucose uptake in muscle fibres and adipocytes, and inhibits gluconeogenesis in the liver [22]. Because isoflavones in soybeans have a chemical structure similar to that of oestrogen, the glucose-lowering effect of isoflavones has attracted attention. Several experimental studies have suggested that isoflavones have anti-obesity or glucose-lowering effects via various biological mechanisms. Genistein inhibits glucose uptake in the intestinal brush border by inhibiting tyrosine kinase activity or decreasing sodium-dependent glucose transport [5]. Isoflavones also have glucose-lowering effects by activating insulin secretion by the islet cells and inhibiting the proliferation of these cells [5]. Isoflavones inhibit adipogenic differentiation by activating AMP-activated protein kinases and lipid accumulation [23-25].

Although a limited number of epidemiological studies have evaluated the association between soybean intake and incidence of type 2 diabetes, our results were consistent with those of previous large cohort studies. In the Shanghai women's health study, the highest quintile for soybean intake exhibited a significantly reduced risk (47\%) of type 2 diabetes compared with the lowest quintile [8]. The Singapore Chinese Health Study reported that the intake of unsweetened soy reduced the risk of type 2 diabetes by up to $30 \%$ [6]. However, evidence on the association between soybean intake and type 2 diabetes is inconsistent. A population-based cohort study in Japan reported that the intake of soybean products and isoflavones was associated with a lower risk of type 2 diabetes in overweight women, but there was no association in men or normal-weight women [9]. In a multiethnic population with relatively low soy food consumption in Hawaii, a higher soy intake was associated with an increased risk of type 2 diabetes in overweight but not in normal-weight people [7].

Although the evidence for a glucose-lowering effect of isoflavones is clear from animal or in vitro studies, the results from epidemiological studies have been inconsistent, and we need to consider these inconsistencies further. First, the inconsistent results may result from a geographical variation in soybean consumption. Isoflavone serum concentrations in Asian populations have been found to be more than ten times higher than those in Western populations, and high levels of consumption among Western populations are far below even the lower levels of intake in our study populations. Second, a measurement error could also give rise to inconsistent results. Previous studies have used FFQs to measure soybean or isoflavone intake. Because FFQs are dependent on the participant's memory, they are generally sensitive to measurement error. In addition, there may be a difference between the dietary intake of a substances and its active level in the blood owing to a variability in food, food-processing and storage, individual variability in uptake and metabolism, and variability of the gut microbiome. Third, the discrepancy between epidemiological studies of soy isoflavones could be attributed to a failure to distinguish between equol producers and nonproducers in the metabolism of isoflavones. Although equol is biotransformed from daidzein by the metabolism caused by gut bacteria, some individuals cannot metabolise daidzein to equol. Up to $80 \%$ of Asian individuals produce equol after consuming soy products, in contrast to only $30-40 \%$ of Western populations [26, 27]. There is increasing evidence that the endocrine-related clinical efficacy of isoflavone may be modified by equol-producing status [28]. However, FFQs cannot distinguish equol producers from equol non-producers.

In our study, isoflavones were associated with type 2 diabetes only in women. This may be explained partially by the role of endogenous sex hormones in the development of type 2 diabetes. Hyperandrogenic conditions have been strongly associated with glucose intolerance and insulin resistance in women, whereas hypoandrogenic conditions have been associated with insulin resistance and adiposity in men $[29,30]$. As oestrogen receptor and androgen pathway modulators, isoflavones have antiandrogenic properties and can act as oestrogen receptor agonists or antagonists depending on endogenous oestrogen levels [31, 32].

In our studies, genistein and equol, but not daidzein and glycitein, were associated with type 2 diabetes. The action of isoflavones may depend on their bioavailability. Compared with the binding activity of $17-\beta$-oestradiol to receptors, the binding activity of genistein was $4-87 \%$ that of $17-\beta$ oestradiol, and daidzein binding was $0.1-0.5 \%$ that of $17-\beta$ oestradiol [33]. Genistein reaches its peak concentration 


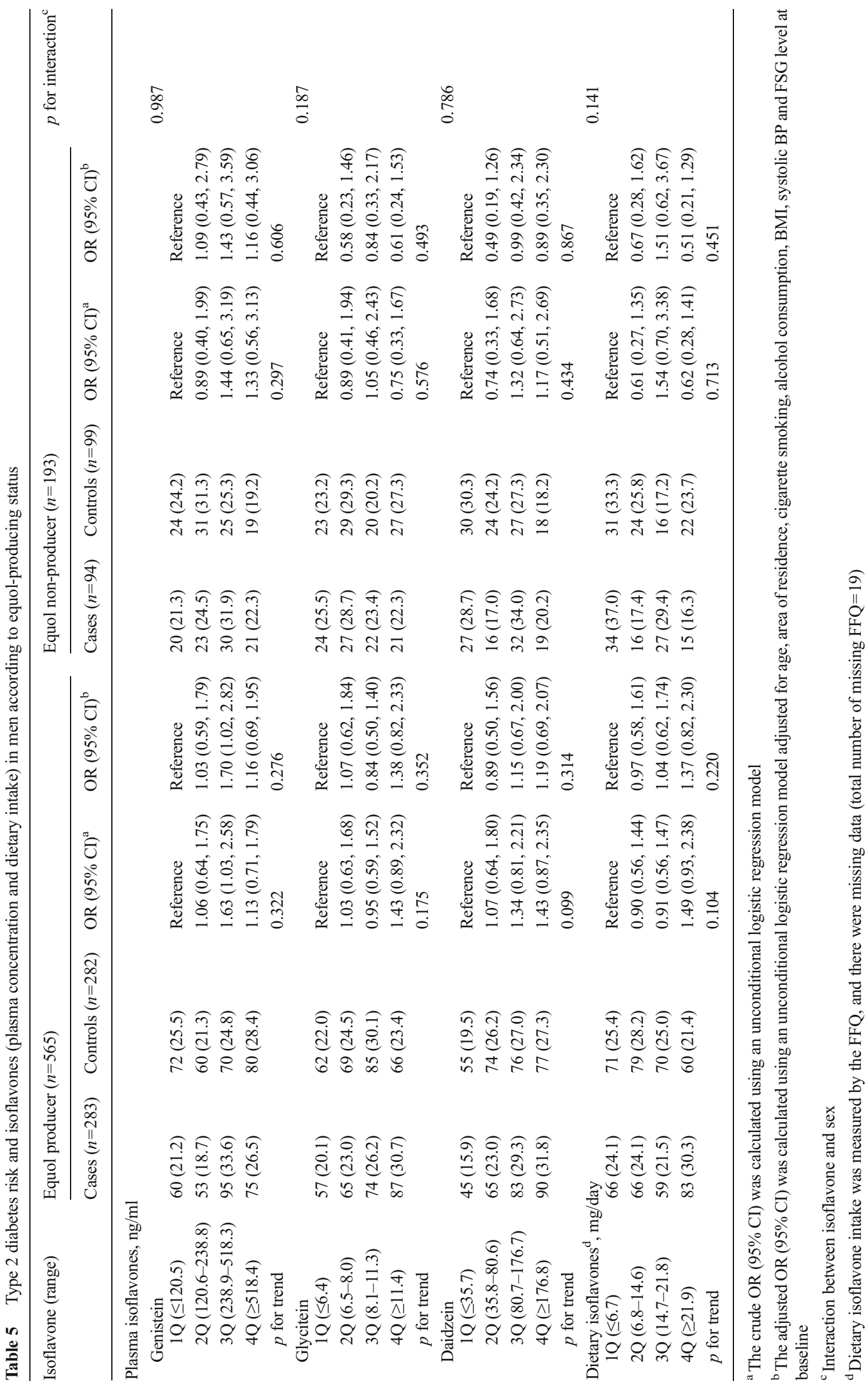


earlier but has a slower plasma clearance rate than daidzein and glycitein [34]. Equol has a slower plasma clearance rate and a longer half-life [11]. Equol also has a 100-fold higher affinity for oestrogen receptors and a higher bioavailability than daidzein and glycitein [35].

Although many studies have reported the role of equolproducing status as an effect-modifier in the association between the intake of soybean products and endocrine-related diseases, the mechanism for this is not clear [36, 37]. Because of equol's low affinity for serum protein, high affinity for the oestrogen receptor and high antioxidant activity, the clinical effectiveness of soy isoflavones may be a function of the ability to biotransform soy isoflavones to the more potent oestrogenic metabolite equol, which may enhance the action of soy isoflavones [38].

Our study has several limitations in its ability to assess the causal relationship between plasma isoflavone concentrations and incidence of type 2 diabetes. One limitation is that we had no information on the type of diabetes. However, incidence rates of type 1 diabetes are extremely low in Asia, and the annual incidence rates of type 1 diabetes in individuals less than 15 years old have been reported as $0.6-2.2$ cases per 100,000 in Korea [39, 40]. Moreover, our study population consisted of individuals over 40 years old. In this population, the incidence of type 1 diabetes is very low; therefore, we considered all our patients to have type 2 diabetes.

Second, because isoflavones have short half-lives in the blood, plasma levels are particularly affected by the time elapsed since the last meal. Nevertheless, we collected samples after overnight fasting so that we could minimise the effect of fluctuations in isoflavone concentration due to different time lapses after food intake.

Third, there was no information on cooking methods. The study by Mueller et al [6] found an inverse association between unsweetened soy products and type 2 diabetes, but no association or a slight positive association for sweetened soy. In the present analysis, however, we could not distinguish unsweetened soy from sweetened soy, although most Korean soybean foods are unsweetened.

Fourth, although loss to follow-up is inevitable in most cohort studies, it can lead to selection bias when loss to follow-up is non-random and is related to both the exposure and the outcome [41]. In our study population, loss to followup was not associated with isoflavone intake measured by the FFQ. Unfortunately, we cannot identify whether loss to follow-up was associated with plasma isoflavone levels and incidence of type 2 diabetes.

Fifth, a dietary intake of soybeans might be correlated with other dietary habits such as the consumption of vegetables, fruit and other healthy foods, and this could act as a confounder. Although we evaluated the dietary intake to assess the confounders, including total energy intake, carbohydrates, vitamin $\mathrm{C}$ and fibre, we could not totally exclude residual potential confounding.

Sixth, the validity of our FFQ to estimate isoflavone intake has not been assessed, although a validation assessment is essential because only a limited number of soy foods can be included in an FFQ. Therefore we encountered limitations in terms of explaining clearly why the results relating to the plasma concentrations of isoflavone and dietary isoflavone intake were different.

Our study also has several strengths. First, the direct measurement of plasma isoflavone concentrations not only provides a metric of intake, but also reflects the results of the absorption and metabolism of isoflavones. Therefore, plasma levels may reflect relevant biological doses more accurately than the FFQ does. Second, the potential information bias was reduced because our study design was prospective and isoflavones were evaluated using prediagnosis plasma samples. Third, by selecting cases and controls from within the same cohort, we could avoid the selection bias that is inherent in case-control studies. Fourth, an OGTT was included in the criteria for the identification of type 2 diabetes. By including an OGTT to make the diagnosis, we identified more cases than we would have based only on FSG or self-reported treatment with hypoglycaemic medication. As a result, the misclassification bias for case identification may have been reduced.

In conclusion, the genistein concentration was associated with a decreased risk of type 2 diabetes in women, and this inverse association between genistein and type 2 diabetes was prominent in equol-producing individuals. A large intervention study is necessary to verify the modifying effect of equol in the association between soybean intake and type 2 diabetes.

Funding This research was supported by a grant (2010-N71001-00) from the Korea Centers for Disease Control and Prevention.

Duality of interest The authors declare that there is no duality of interest associated with this manuscript.

Contribution statement KPK contributed to the conception and design of the manuscript, the interpretation of data and the drafting of the article. CSK, YJK and JKP contributed to the acquisition of data and critical revision of the manuscript. YA and SJP contributed to the conception and design of the manuscript, acquisition and interpretation of the data, and revising the article. YKL, KYY and SSK contributed to the conception and design of the manuscript and critically revised it for important intellectual content. All authors approved the final manuscript. SSK is the guarantor of this work.

\section{References}

1. Wild S, Roglic G, Green A, Sicree R, King H (2004) Global prevalence of diabetes: estimates for the year 2000 and projections for 2030. Diabetes Care 27:1047-1053 
2. Meyer MR, Clegg DJ, Prossnitz ER, Barton M (2011) Obesity, insulin resistance and diabetes: sex differences and role of oestrogen receptors. Acta Physiol 203:259-269

3. Jayagopal V, Albertazzi P, Kilpatrick ES et al (2002) Beneficial effects of soy phytoestrogen intake in postmenopausal women with type 2 diabetes. Diabetes Care 25:1709-1714

4. Bhathena SJ, Velasquez MT (2002) Beneficial role of dietary phytoestrogens in obesity and diabetes. Am J Clin Nutr 76:11911201

5. Vedavanam K, Srijayanta S, O'Reilly J, Raman A, Wiseman H (1999) Antioxidant action and potential antidiabetic properties of an isoflavonoid-containing soyabean phytochemical extract (SPE). Phytother Res PTR 13:601-608

6. Mueller NT, Odegaard AO, Gross MD et al (2012) Soy intake and risk of type 2 diabetes mellitus in Chinese Singaporeans: soy intake and risk of type 2 diabetes. Eur J Nutr 51:1033-1040

7. Morimoto Y, Steinbrecher A, Kolonel LN, Maskarinec G (2011) Soy consumption is not protective against diabetes in Hawaii: the Multiethnic Cohort. Eur J Clin Nutr 65:279-282

8. Villegas R, Gao YT, Yang G et al (2008) Legume and soy food intake and the incidence of type 2 diabetes in the Shanghai Women's Health Study. Am J Clin Nutr 87:162-167

9. Nanri A, Mizoue T, Takahashi Y et al (2010) Soy product and isoflavone intakes are associated with a lower risk of type 2 diabetes in overweight Japanese women. J Nutr 140:580-586

10. Rowland IR, Wiseman H, Sanders TA, Adlercreutz H, Bowey EA (2000) Interindividual variation in metabolism of soy isoflavones and lignans: influence of habitual diet on equol production by the gut microflora. Nutr Cancer 36:27-32

11. Setchell KD, Brown NM, Lydeking-Olsen E (2002) The clinical importance of the metabolite equol - a clue to the effectiveness of soy and its isoflavones. J Nutr 132:3577-3584

12. Perera FP, Weinstein IB (2000) Molecular epidemiology: recent advances and future directions. Carcinogenesis 21:517-524

13. Liggins J, Bluck LJ, Runswick S, Atkinson C, Coward WA, Bingham SA (2000) Daidzein and genistein contents of vegetables. Br J Nutr 84:717-725

14. Shin C, Abbott RD, Lee H, Kim J, Kimm K (2004) Prevalence and correlates of orthostatic hypotension in middle-aged men and women in Korea: the Korean Health and Genome Study. J Hum Hypertens $18: 717-723$

15. Cho N, Joo S, Kim J, Abbott RD, Kimm K, Shin C (2006) Relation of habitual snoring with components of metabolic syndrome in Korean adults. Diabetes Res Clin Pract 71:256-263

16. Ahn Y, Kwon E, Shim JE et al (2007) Validation and reproducibility of food frequency questionnaire for Korean genome epidemiologic study. Eur J Clin Nutr 61:1435-1441

17. Park MK, Song Y, Joung H, Li SJ, Paik HY (2007) Establishment of an isoflavone database for usual Korean foods and evaluation of isoflavone intake among Korean children. Asia Pac J Clin Nutr 16: $129-139$

18. Grace PB, Taylor JI, Botting NP et al (2003) Quantification of isoflavones and lignans in serum using isotope dilution liquid chromatography/tandem mass spectrometry. Rapid Commun Mass Spectrom RCM 17:1350-1357

19. Fujimoto K, Tanaka M, Hirao Y et al (2008) Age-stratified serum levels of isoflavones and proportion of equol producers in Japanese and Korean healthy men. Prostate Cancer Prostatic Dis $11: 252-257$

20. Hong KW, Ko KP, Ahn Y et al (2012) Epidemiological profiles between equol producers and nonproducers: a genomewide association study of the equol-producing phenotype. Genes Nutr 7:567-574

21. Akaza H, Miyanaga N, Takashima N et al (2004) Comparisons of percent equol producers between prostate cancer patients and controls: case-controlled studies of isoflavones in Japanese, Korean and American residents. Jpn J Clin Oncol 34:86-89

22. Louet JF, LeMay C, Mauvais-Jarvis F (2004) Antidiabetic actions of estrogen: insight from human and genetic mouse models. Curr Atheroscler Rep 6:180-185

23. Hwang JT, Park IJ, Shin JI et al (2005) Genistein, EGCG, and capsaicin inhibit adipocyte differentiation process via activating AMP-activated protein kinase. Biochem Biophys Res Commun 338:694-699

24. Kim HK, Nelson-Dooley C, Della-Fera MA et al (2006) Genistein decreases food intake, body weight, and fat pad weight and causes adipose tissue apoptosis in ovariectomized female mice. J Nutr 136: 409-414

25. Park HJ, Della-Fera MA, Hausman DB, Rayalam S, Ambati S, Baile CA (2009) Genistein inhibits differentiation of primary human adipocytes. J Nutr Biochem 20:140-148

26. Morton MS, Arisaka O, Miyake N, Morgan LD, Evans BA (2002) Phytoestrogen concentrations in serum from Japanese men and women over forty years of age. J Nutr 132:3168-3171

27. Lampe JW, Karr SC, Hutchins AM, Slavin JL (1998) Urinary equol excretion with a soy challenge: influence of habitual diet. Proc Soc Exp Biol Med 217:335-339

28. Shor D, Sathyapalan T, Atkin SL, Thatcher NJ (2012) Does equol production determine soy endocrine effects? Eur J Nutr 51:389-398

29. Andersson B, Marin P, Lissner L, Vermeulen A, Bjorntorp P (1994) Testosterone concentrations in women and men with NIDDM. Diabetes Care 17:405-411

30. Oh JY, Barrett-Connor E, Wedick NM, Wingard DL (2002) Endogenous sex hormones and the development of type 2 diabetes in older men and women: the Rancho Bernardo study. Diabetes Care 25:55-60

31. Cornwell T, Cohick W, Raskin I (2004) Dietary phytoestrogens and health. Phytochemistry 65:995-1016

32. Ford ES, Liu S (2005) Invited commentary: acne in adolescenceprotecting the heart but damaging the prostate later in life? Am J Epidemiol 161:1102-1106

33. Kuiper GG, Carlsson B, Grandien K et al (1997) Comparison of the ligand binding specificity and transcript tissue distribution of estrogen receptors alpha and beta. Endocrinology 138:863-870

34. Shelnutt SR, Cimino CO, Wiggins PA, Ronis MJ, Badger TM (2002) Pharmacokinetics of the glucuronide and sulfate conjugates of genistein and daidzein in men and women after consumption of a soy beverage. Am J Clin Nutr 76:588-594

35. Sathyamoorthy N, Wang TT (1997) Differential effects of dietary phyto-oestrogens daidzein and equol on human breast cancer MCF-7 cells. Eur J Cancer 33:2384-2389

36. Fuhrman BJ, Teter BE, Barba M et al (2008) Equol status modifies the association of soy intake and mammographic density in a sample of postmenopausal women. Cancer Epidemiol Biomark Prev 17:3342

37. Wu J, Oka J, Ezaki J et al (2007) Possible role of equol status in the effects of isoflavone on bone and fat mass in postmenopausal Japanese women: a double-blind, randomized, controlled trial. Menopause 14:866-874

38. Yuan JP, Wang JH, Liu X (2007) Metabolism of dietary soy isoflavones to equol by human intestinal microflora-implications for health. Mol Nutr Food Res 51:765-781

39. Ko KW, Yang SW, Cho NH (1994) The incidence of IDDM in Seoul from 1985 to 1988 . Diabetes Care 17:1473-1475

40. Matsuura N, Ko KW, Park YS, Elliott R (1996) Molecular epidemiology of IDDM in the Western Pacific Rim Region. WHO DiaMond Molecular Epidemiology Sub-Project Group. Diabetes Res Clin Pract 34(Suppl):S117-S123

41. Greenland S (1977) Response and follow-up bias in cohort studies. Am J Epidemiol 106:184-187 